\title{
Effects of a loop array layer on a micro-inductor for future RF MEMS Components
}

\author{
Charles-Marie Tassetti, Gaëlle Lissorgues, *Jean-Paul Gilles \\ Groupe ESIEE, Cité Descartes, 93162 Noisy-le-Grand, France \\ Tel : +33 (0) 1459266 96, Email : tassettc@esiee.fr, g.lissorgues@esiee.fr \\ *IEF, Université Paris Sud, 91405 Orsay, France \\ Tel : +33 (0) 1691565 85, Email : jean-paul.gilles@ief.u-psud.fr
}

\begin{abstract}
Tunable micro-inductors can be designed controlling magnetic coupling coefficient. This paper presents the inductance variation produced by loops strongly magnetically coupled with a micro-inductor. A compact lumped element model, based on the geometry, gives result in good agreement with measurements in the RF frequency range. Then, this model will be applied to the study of the tunable MEMS inductor described below.
\end{abstract}

\section{INTRODUCTION}

Tunable micro-inductors can be designed using magnetic materials. The circuit inductance increases with the effective permeability of the surrounding environment. Some magnetic materials can be driven by a small DC magnetic field applied in their hard direction, and resulting in a permeability variation. Our work is to design tunable micro-inductors for microwave communication systems. But at this frequency range, over $1 \mathrm{GHz}$, most of magnetic materials turn into non-magnetic materials, with a relative permeability decreasing to $\mu_{\mathrm{r}}=1$. Our idea is to create a kind of artificial magnetic layer with a "loop array". Loops will change the magnetic flow through the micro-inductor. Recent papers [1] [2] use a similar method, and patterned ground shield, to increase the quality factor of micro-inductors. In our case, we are interested in the inductance tuning: we present a method to compute the inductance variation produced by the loop array, and then report some measurements on laboratory structures.

\section{ANALYSIS METHOD}

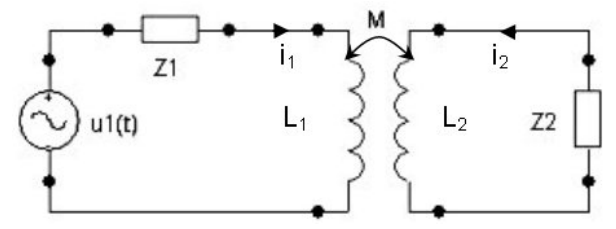

Fig.1. Micro-inductor / loop circuit model.
The method described below is a "circuit" method, so it is not as rigorous as a field theory analysis. It is based on the transformer equations, and it takes into account all the coupling coefficients between micro-inductor / loops and loops / loops. In a transformer, there are two circuits: for our application, the primary is the micro-inductor, and one loop represents the secondary, Fig1. These two circuits are magnetically coupled creating a mutual inductance $M$. A $\mathrm{RF}$ signal $u_{1}(t)$ is flowing through the micro-inductor producing an induced current in the secondary.

\section{A. Theory}

In our design, the loop representing the secondary circuit is in short-circuit configuration, and finally the system resolution gives the expression of the primary circuit equivalent impedance (1).

$$
Z_{\text {eq }}(\omega)=\left(Z_{1}+j L_{1} \omega\right)+\frac{M^{2} \omega^{2}}{\left(Z_{2}+j L_{2} \omega\right)}
$$

This linear system can be written into a matrix formulation, $[\mathrm{U}]=[\mathrm{z}] .[\mathrm{I}]$, and then extended to the resolution of a $n$ loops configuration. The impedance matrix is the impedance of each loop and takes into account all the coupling factors. When an arbitrary value for $\mathrm{u}_{1}(\mathrm{t})$ is set, the current matrix is $[\mathrm{r}]=[\mathrm{z}]^{-1}$. [u $]$.

\section{B. Application to loop array layers}

This method can model substrates made of a high number of loops, on several levels, Fig. 2. Loops are located by their center coordinates. If the substrate is created by a one level $n^{*} n$ loop array layer, we have $\mathrm{n}^{2}$ loops underneath the micro-inductor, then, the total inductance number to compute is $n^{2}+1$, and $n^{2}\left(n^{2}+1\right)$ mutual inductances. To simplify the operation, we can use identical loops, it reduces the inductance number to 2 . Moreover, using geometrical symmetries, Fig. 3, is a good way to minimize the mutual inductance number. All the 
mutual inductors are a function of the distance separating their center. Each loop is indexed by a line number $i$ and a row number $j$. The mutual inductor between loop $(i, j)$ and $(k, l)$ is $M_{m, n}, \quad m=|i-k|$ et $n=|j-l|$. Only the mutual inductances between loop $(0,0)$ and loops represented on Fig.3 are necessary to be computed when considering symmetries.
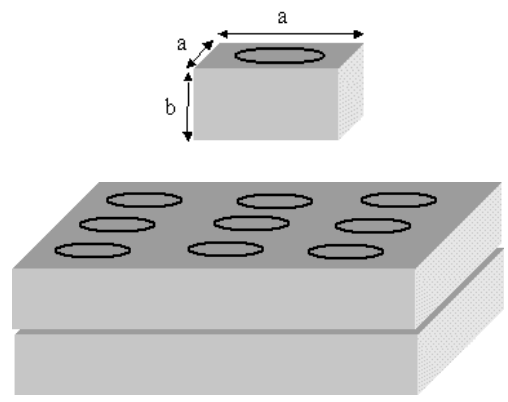

Fig.2.Basic cell and substrate.

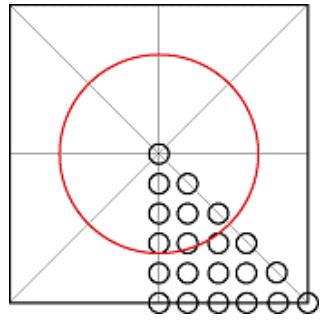

Fig.3.Example of the system symmetries.

Symmetries drastically reduce the computational time. For several loop layers, the method is quite the same, except we have to add the inter-layer magnetic coupling phenomena.

\section{Electric network}

For a $n^{2}$ loops layer, the impedance is a symmetric $\left(n^{2}+1\right) *\left(n^{2}+1\right)$ matrix:

$$
[Z]=\left(\begin{array}{cccc}
Z_{1}+j L_{1} \omega & j M_{2,1} \omega & \ldots & j M_{n, 1} \omega \\
j M_{2,1} \omega & Z_{2}+j L_{2} \omega & \ddots & \vdots \\
\vdots & \ddots & \ddots & j M_{n, n-1} \omega \\
j M_{n, 1} \omega & \ldots & j M_{n, n-1} \omega & Z_{2}+j L_{2} \omega
\end{array}\right)(4)
$$

Finally, the equivalent impedance is $Z_{e q}(\omega)=u_{1} / i_{1}$.

\section{Results}

Some layer configurations have been tested using this method, the computed equivalent inductance of a $1.5 \mathrm{~mm}$ radius micro-inductor is shown in Fig. 4, loops and micro- inductor are separated by a $5000 \AA$ gap. There is only one loop array layer, $a$ is the basic cell length, Fig.2:
(1): $n=5 \quad a=800 \mu \mathrm{m}$
(2): $n=10 \quad a=550 \mu \mathrm{m}$
(3): $n=13 \quad a=350 \mu \mathrm{m}$
(4): $n=5 \quad a=800 \mu \mathrm{m}$
loop radius $=100 \mu \mathrm{m}$ loop radius $=200 \mu \mathrm{m}$ loop radius $=100 \mu \mathrm{m}$ loop radius $=300 \mu \mathrm{m}$

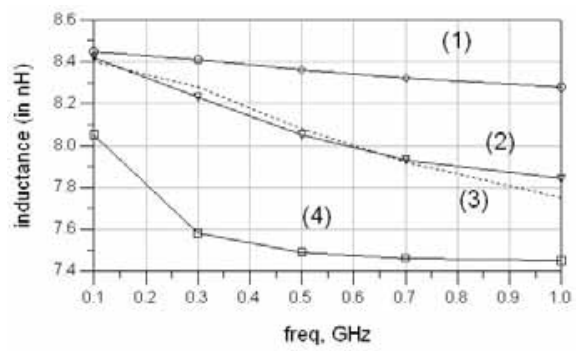

Fig.4. Circuit equivalent inductance versus frequency for different geometrical configurations.

This method has neglected the capacitive coupling effect between loops and micro-inductor. Moreover, to reduce the resolution time, the mutual inductances are computed by the Neumann's formula, which has a low accuracy for small dimensions.

\section{LOOP ARRAY DESING AND REALIZATION}

\section{A. Experimental realization}

Processed inductors are shown on Fig.5. The microinductance (a) has a radius of $1.5 \mathrm{~mm}$ with a $50 \mu \mathrm{m}$ width line. All the micro-loops have a $50 \mu \mathrm{m}$ width line. The different dimensions are:

(b) : $13 * 13100 \mu \mathrm{m}$ radius loop array

(c) $: 5 * 5300 \mu \mathrm{m}$ radius loop array

(d) : $84100 \mu \mathrm{m}$ loops

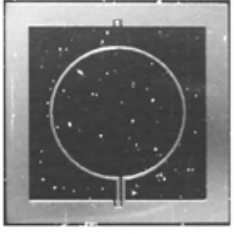

(a)

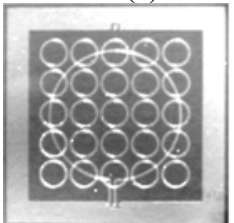

(c)

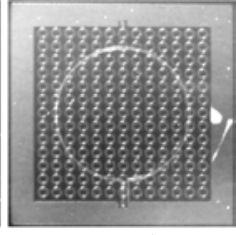

(b)

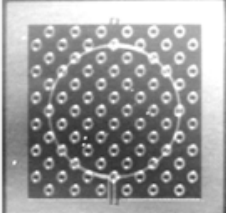

(d)
Fig.5. Micro-inductors realized with different loops arrays, using poor accuracy masks for this process, resulting in large dimensions for RF measurements. 


\section{B. Measurements}

We extract the equivalent inductance value in each configuration, Fig. 6 and Table I. The ratio between the (a) micro-inductance value and with a loop array underneath is constant over the range $300-800 \mathrm{MHz}$. For example, the ratio $\mathrm{L}(\mathrm{a}) / \mathrm{L}(\mathrm{c})$ is around 1.3 , corresponding to a $30 \%$ inductance variation. We can also notice that the resonance frequency is higher in (d) configuration, due to fewer parasitic capacitance effects, while the tuning ratio is lower.

TABLE I

EXTRACTED EQUIVALENT INDUCTANCE $(\mathrm{nH})$

\begin{tabular}{|l|l|l|l|l|l|}
\multicolumn{1}{c|}{} & $\mathbf{0 . 1 G H z}$ & $\mathbf{0 . 3 G H Z}$ & $\mathbf{0 . 5 G H z}$ & $\mathbf{0 . 7 G H z}$ & $\mathbf{1 G H z}$ \\
\hline (a) & 8.41 & 8.45 & 8.49 & 8.52 & 8.67 \\
\hline (b) & 5.7 & 6.56 & 6.64 & 6.62 & 6.49 \\
\hline (c) & 5.53 & 6.43 & 6.45 & 6.37 & 6.35 \\
\hline (d) & 6.77 & 7.26 & 7.35 & 7.5 & 7.69 \\
\hline
\end{tabular}

\section{Conclusions}

These first measurements are very promising because the micro-inductor variation ratio is significant without any optimization of the design. These structures could be used over a wide frequency range (towards $10 \mathrm{GHz}$ and more) if all geometrical dimensions are reduced. A FET can be associated with each loop to control it (open/shortcircuit configuration), then we can have discrete inductance values.

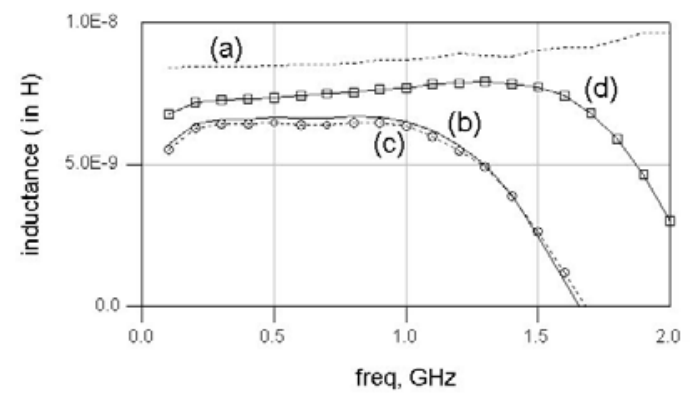

Fig.6. Measured inductance versus frequency.

\section{CONTINUOUS VARIATIONS}

In order to have high continuous variation ratios, we need great mechanical displacements between the microinductor and loops. As the magnetic coupling coefficient is a function of the distance separating the micro-inductor and the loop, great mechanical displacements will produce high variation ratios. Electrostatic actuation is the easiest way to have mechanical displacements, however it requires high voltage. Some of our structures have more than $15 \mu \mathrm{m}$ gap, and $150 \mathrm{~V}$ pull-in voltage. In our design the loop and the microinductor have their center aligned on the same axe, mechanical displacements can only occur along the $\mathbf{z}$ axe, Fig. 7.

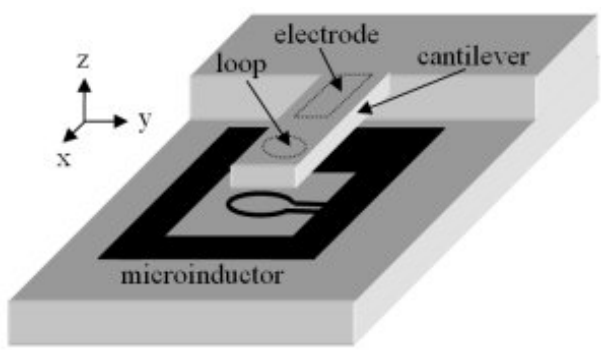

Fig. 7. 3D view (loop and electrode on the cantilever are shown by transparency)

\section{A. Measurement setup}

The microwave on-wafer test measurement setup is: Agilent 8722ES vector network analyzer using groundsignal-ground probes with a $150 \mu \mathrm{m}$ pitch, performed between 0.1 to $5 \mathrm{GHz}$. One port of the inductor being connected to ground, we make reflexion measurements $\left(\mathrm{S}_{11}\right.$ parameter). We need two more probes for $\mathrm{DC}$ actuation polarization: the first one is connected to the RF ground line and the second one to the cantilever electrode pad, Fig.8. We measure the structures at different actuation voltages, from $0 \mathrm{~V}$ to $150 \mathrm{~V}$, which is the cantilever pull-in voltage.

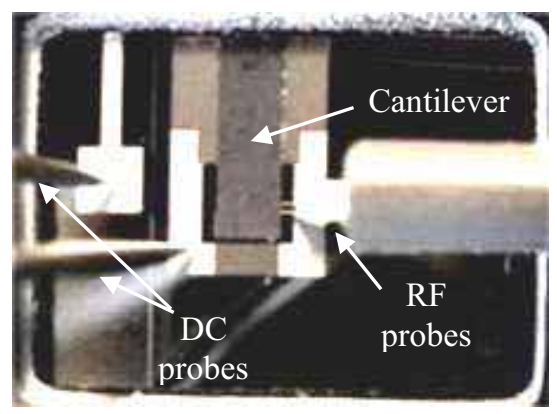

Fig. 8. Measurement setup: actuation probes and RF probes (Top Side)

\section{B.Results and extracted parameters}

In order to evaluate the inductance variation, we can simply extract this inductance value from $Z_{11}$, $\mathrm{L}_{\mathrm{eq}}=\operatorname{Im}\left(\mathrm{Z}_{11}\right) / \omega$.

Previously we associated equation (1) to the analysis of our system. We can define two different domains: the first one in the low frequency range, and the second one in the high frequency range, Table II. At low frequencies the resistor impedance is prevailing, so we have an inductance value which is frequency dependent. At higher frequencies, the inductor impedance is greater than the resistor impedance and the inductance value becomes constant. For structure (c), Fig.9, inductance value becomes constant at $1 \mathrm{GHz}$. 
TABLE II

TWO MAIN DOMAINS

\begin{tabular}{cc}
\hline \hline Low frequencies & High frequencies \\
\hline $\mathrm{R}>>\mathrm{L}_{0} \omega$ & $\mathrm{L}_{0} \omega>>\mathrm{R}$ \\
$\mathrm{L}_{\mathrm{eq}} \approx \mathrm{L}_{0} \cdot\left[1-\left(\frac{k \cdot \mathrm{L}_{0} \omega}{\mathrm{R}}\right)^{2}\right]$ & $\mathrm{L}_{\mathrm{eq}} \approx \mathrm{L}_{0} \cdot\left(1-k^{2}\right)$ \\
\hline \hline
\end{tabular}

Extracted inductance values are the proof that we can reach very high variation ratios by optimizing our tunable microinductor design. From $1 \mathrm{GHz}$ to $5 \mathrm{GHz}$, structure (c) has a $0.97 \mathrm{nH}$ microinductor at $0 \mathrm{~V}$ and $0.71 \mathrm{nH}$ for a $150 \mathrm{~V}$ actuation voltage: it makes a $36 \%$ variation ratio (for some structure we can double the inductance value, 0.5nH@5GHz and 1nH @ $1 \mathrm{GHz}$ ).

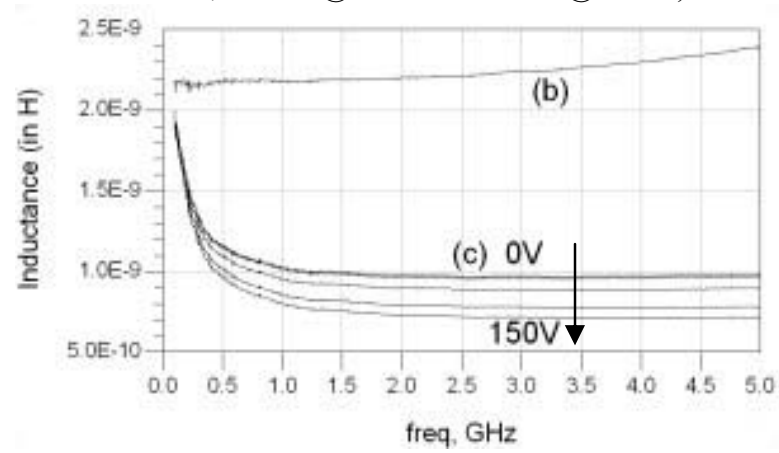

Fig. 9. Plot of the inductance variation for structure (c) with actuation voltage from 0 to $150 \mathrm{~V}$

\section{C.Equivalent circuit}

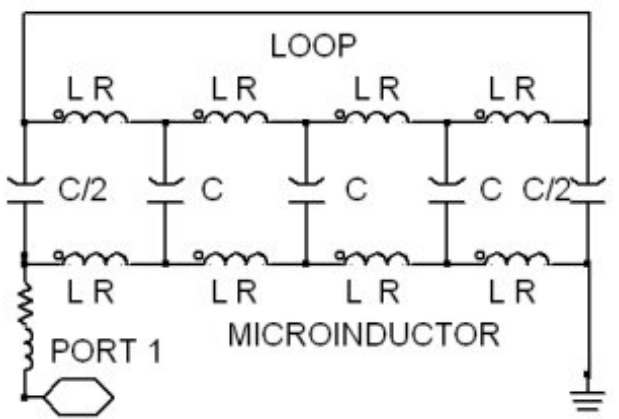

Fig. 10. Equivalent circuit : for (c) at $150 \mathrm{~V}, \mathrm{R}=0.72 \Omega$, $\mathrm{L}=0.51 \mathrm{nH}, \mathrm{C}=0.1 \mathrm{pF}, \mathrm{k}=0.8$.

We built an equivalent circuit based on the design geometry. Simulations of the compact model, Fig. 10, give results in good agreement with measurements, Fig. 11. Elements of this model can be evaluated by classical equations: the inductance is computed with a magnetic flow method as well as the coupling factor. Wire thickness is less than the skin depth at $5 \mathrm{GHz}$, so the wire resistance is the DC resistance, and the capacitance between the inductor and the loop is approximated by the "parallel- plates formula". We only use magnetic coupling between two adjacent wires, and all other coupling coefficients are neglected. The equivalent circuit for structure (c) at $150 \mathrm{~V}$ is $\mathrm{R}=0.72 \Omega, \mathrm{L}=0.51 \mathrm{nH}$ for each branches, $\mathrm{C}=0.1 \mathrm{pF}$ and $k=0.84$.

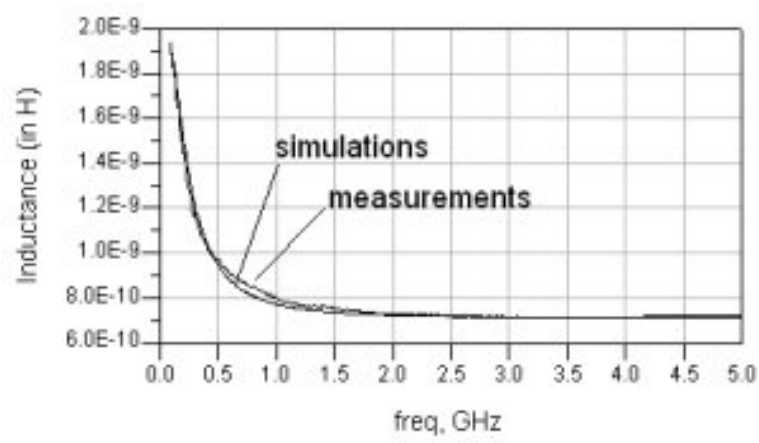

Fig. 11 : Comparison between (c) structure at $150 \mathrm{~V}$ extracted inductance and equivalent circuit inductance.

\section{PERSPECTIVES AND CONCLUSION}

Tunable micro-inductors can be designed using an "artificial" magnetic layer with a loop array. Micro-loops can reduce the inductance of a micro-inductor by $30 \%$ and this technique is well suitable for frequencies over $1 \mathrm{GHz}$. This variation ratio is function of the gap between the micro-inductor and the loop array. This gap could be, for example, increased or decreased by mechanical displacements in an RF MEMS structure, resulting in an inductance variation. The feasibility of such a tunable micro-inductor, with continuous variations, has been presented. It is possible to double the inductance value. The integration on silicon of tunable capacitors and tunable inductors together will offer new possibilities in wideband filter architectures or new matching solutions above $1 \mathrm{GHz}$.

\section{ACKNOWLEDGEMENT}

The authors wish to acknowledge the assistance and support of the SMM team during the fabrication process in clean room at ESIEE. This work is supported by Thales Airborne Systems and the French Ministry Of Defense.

\section{REFERENCES}

[1] H-S. Wu, C-K. C. Tzuang, "PBG-enhanced Inductor," IEEE MTT-S Symp. Dig., June 2002.

[2] J-H. Chang, Y-S. Youn, H-K. Yu, C-K. Kim, "Effects of Dummy patterns and Substrate on Spiral Inductors for Sub-micron RF ICs," IEEE MTT-S Symp. Dig., June 2002. 